\title{
A general structure theorem for the nash equilibrium correspondence
}

Citation for published version (APA):

Predtetchinski, A. (2004). A general structure theorem for the nash equilibrium correspondence.

METEOR, Maastricht University School of Business and Economics. METEOR Research Memorandum No. 023 https://doi.org/10.26481/umamet.2004023

Document status and date:

Published: 01/01/2004

DOI:

10.26481/umamet.2004023

Document Version:

Publisher's PDF, also known as Version of record

\section{Please check the document version of this publication:}

- A submitted manuscript is the version of the article upon submission and before peer-review. There can be important differences between the submitted version and the official published version of record.

People interested in the research are advised to contact the author for the final version of the publication, or visit the DOI to the publisher's website.

- The final author version and the galley proof are versions of the publication after peer review.

- The final published version features the final layout of the paper including the volume, issue and page numbers.

Link to publication

\footnotetext{
General rights rights.

- You may freely distribute the URL identifying the publication in the public portal. please follow below link for the End User Agreement:

www.umlib.nl/taverne-license

Take down policy

If you believe that this document breaches copyright please contact us at:

repository@maastrichtuniversity.nl

providing details and we will investigate your claim.
}

Copyright and moral rights for the publications made accessible in the public portal are retained by the authors and/or other copyright owners and it is a condition of accessing publications that users recognise and abide by the legal requirements associated with these

- Users may download and print one copy of any publication from the public portal for the purpose of private study or research.

- You may not further distribute the material or use it for any profit-making activity or commercial gain

If the publication is distributed under the terms of Article $25 \mathrm{fa}$ of the Dutch Copyright Act, indicated by the "Taverne" license above, 


\title{
A General Structure Theorem for the Nash Equilibrium Correspondence
}

\author{
Arkadi Predtetchinski ${ }^{* \dagger}$
}

May 25, 2004

\begin{abstract}
I consider $n$-person normal form games where the strategy set of every player is a non-empty compact convex subset of Euclidean space, and the payoff function of player $i$ is continuous and concave in player $i$ 's own strategies. No further restrictions (such as multilinearity of the payoff functions or the requirement that the strategy sets be polyhedral) are imposed. In this setting we demonstrate that the graph of the Nash equilibrium correspondence is homeomorphic to the space of games. This result generalizes a well-known structure theorem of Kohlberg and Mertens [6].
\end{abstract}

JEL classification codes: C72.

Keywords: Nash equilibrium correspondence.

*Department of Economics, Maastricht University, P.O. Box 616, 6200 MD Maastricht, The Netherlands. E-mail: a.predtetchinski@algec.unimaas.nl

${ }^{\dagger}$ Helpful comments of Jean-Jacques Herings and Hans Peters are gratefully acknowledged. 


\section{Introduction}

The study of topological properties of the graphs of various equilibrium correspondences is an important ingredient of present-day equilibrium analysis. It provides a unified treatment of such topics as existence, optimality, (in)determinateness of equilibrium and dynamics of adjustment processes. The classical work of Balasko [1] serves an excellent illustration of how the properties of the equilibrium manifold can be used to approach some traditional problems in general equilibrium theory. Furthermore, the study of equilibrium manifolds is of a considerable independent interest.

In Kohlberg and Mertens [6, Theorem 1] it is shown that the graph of the Nash equilibrium correspondence in normal form games is homeomorphic to Euclidean space. In the context of a pure exchange economy, Balasko [1] shows that the graph of the Walrasian equilibrium correspondence is homeomorphic to Euclidean space. A topological characterization of the pseudo-equilibrium manifold in economies with incomplete markets is given in Zhou [8]. Related references are Blume and Zame [2] and Govindan and Wilson [5] where the semi-algebraic nature of various game-theoretic solution concepts is established, and Demichelis and Germano [3, 4] who focus at the unknottedness property of equilibrium manifolds.

This paper contributes to the literature above by establishing the analogue of the Kohlberg and Mertens' [6] structure theorem in a very general setup. I consider the space of normal form games as parameterized by payoff functions. It is assumed that the strategy set of every player is a non-empty compact convex set of Euclidean space, and the payoff function of player $i$ is continuous and concave in player $i$ 's own strategies. No further restrictions (such as multilinearity of the payoff functions or the requirement that the strategy sets be polyhedral) are imposed. In this setting it is demonstrated that the graph of the Nash equilibrium correspondence is homeomorphic to the space of games. In addition, I show that there exists a homeomorphism that preserves the multilinearity in the payoff functions. The result of Kohlberg and Mertens [6] then follows as a corollary to this structure theorem.

The organization of the paper is as follows. In Section 2 we review some mathematical concepts needed for the proof of the structure theorem. As a motivation for the proof, we first establish our result for one-player games. The analysis of this special case is presented in Section 3. In Section 4 we review the structure theorem of Kohlberg and Mertens [6]. The general structure theorem is established in Section 5.

\section{Preliminaries}

In this section we introduce the notation and review some mathematical concepts used in the proof of the structure theorem.

The subdifferential and the normal vectors. Given two vectors $x$ and $y$ of $\mathbb{R}^{n}$ we let $\langle x, y\rangle$ denote the scalar product of $x$ and $y$. Let $f: \mathbb{R}^{n} \longrightarrow \mathbb{R}$ be a concave function, and 
$x$ be a point of $\mathbb{R}^{n}$. A vector $x^{*}$ in $\mathbb{R}^{n}$ is a subgradient of the function $f$ at the point $x$ if

$$
f(z) \leq f(x)+\left\langle z-x, x^{*}\right\rangle \quad \forall z \in \mathbb{R}^{n} .
$$

The set of all subgradients of $u$ at $x$ is called a subdifferential of $u$ at $x$ and is denoted by $\partial u(x)$. By Theorem 23.8 from Rockafellar [7, p. 223], for any two concave functions $f, \bar{f}: \mathbb{R}^{n} \longrightarrow \mathbb{R}$, the subdifferential of the sum is equal to the sum of the subdifferentials:

$$
\partial(f+\bar{f})(z)=\partial f(z)+\partial \bar{f}(z) .
$$

Let $C$ be a compact convex set of $\mathbb{R}^{n}$ and $x$ be a point of $C$. The vector $x^{*}$ in $\mathbb{R}^{n}$ is a normal vector to the set $C$ at the point $x$ if

$$
\left\langle z-x, x^{*}\right\rangle \leq 0 \quad \forall z \in C .
$$

The set of all normal vectors to the set $C$ at the point $x$ is denoted by $N_{x}[C]$. The normal bundle of the set $C$ is a subspace of $C \times \mathbb{R}^{n}$ defined as

$$
N[C]=\left\{\left(x^{*}, x\right) \in \mathbb{R}^{n} \times C \mid x^{*} \in N_{x}[C]\right\} .
$$

By Theorem 27.4 from Rockafellar [7, p. 270], the point $x$ is the maximum of the concave function $f$ on the set $C$ if and only if $\partial u(x) \cap N_{x}[C]$ is a non-empty set.

The compact open topology. Let $X$ and $Y$ be topological spaces, and let $\mathscr{F}(X, Y)$ be the set of all continuous functions from $X$ to $Y$. The compact open topology on $\mathscr{F}(X, Y)$ is defined by the subbase consisting of all sets of the form $\{f \in \mathscr{F}(X, Y): f[E] \subset U\}$, where $E$ is a compact set of $X$ and $U$ is an open set of $Y$. Below we list some properties of the compact open topology.

Suppose that $X$ is a locally compact Hausdorff space. Then the evaluation map $e$ : $\mathscr{F}(X, Y) \times X \longrightarrow Y$ defined by $(f, x) \mapsto f(x)$, is continuous with respect to the compact open topology.

Suppose that $X$ is a locally compact Hausdorff space. Consider a mapping $f: Z \times X \longrightarrow$ $Y$ such that $f_{z}=f(z, \cdot): X \longrightarrow Y$ is a continuous function for each $z \in Z$. Associated with $f$ is the mapping $\phi: Z \longrightarrow \mathscr{F}(X, Y)$ that carries the point $z$ into the function $f_{z}$. Then the mapping $\phi$ is continuous with respect to the compact open topology on $\mathscr{F}(X, Y)$ if and only if $f$ is a continuous mapping.

The mapping

$$
\begin{gathered}
\mathscr{F}\left(\mathbb{R}^{n}, \mathbb{R}^{k}\right) \times \mathscr{F}\left(\mathbb{R}^{n}, \mathbb{R}^{k}\right) \longrightarrow \mathscr{F}\left(\mathbb{R}^{n}, \mathbb{R}^{k}\right) \\
(f, g) \mapsto f+g
\end{gathered}
$$

and the mapping

$$
\begin{gathered}
\mathscr{F}\left(\mathbb{R}^{n}, \mathbb{R}^{k}\right) \times \mathscr{F}\left(\mathbb{R}^{k}, \mathbb{R}^{m}\right) \longrightarrow \mathscr{F}\left(\mathbb{R}^{n}, \mathbb{R}^{m}\right) \\
(f, g) \mapsto g \circ f
\end{gathered}
$$

are both continuous with respect to the compact open topology.

Let $C$ be a non-empty compact convex set of $\mathbb{R}^{n}$, and let $\mathscr{S}\left(\mathbb{R}^{n}, \mathbb{R}\right)$ be the space of all continuous strictly concave functions on $\mathbb{R}^{n}$. Given an $f \in \mathscr{S}\left(\mathbb{R}^{n}, \mathbb{R}\right)$, let $s(f)$ denote the unique point of $C$ where the function $f$ attains its maximum. Then the mapping $\mathscr{S}\left(\mathbb{R}^{n}, \mathbb{R}\right) \longrightarrow \mathbb{R}^{n}$ that carries the function $f$ to the point $s(f)$ is continuous with respect to the compact open topology. 


\section{The one-player case}

Let $X$ be a non-empty compact convex set of the Euclidean space $\mathbb{R}^{k}$. Let $\mathscr{F}$ be the space of all continuous concave functions $f: \mathbb{R}^{k} \longrightarrow \mathbb{R}$ endowed with the compact open topology.

In this section we consider one-player games where the player's strategy set is $X$ and his payoff function is an element $f$ of the space $\mathscr{F}$. A Nash equilibrium of the game $f$ is a strategy that maximizes the payoff function $f$ on the set $X$. The Nash equilibrium correspondence assigns to each $f \in \mathscr{F}$ the set of Nash equilibria of the game $f$. Thus the graph of the Nash equilibrium correspondence is the subspace

$$
\mathscr{N}=\{(f, x) \in \mathscr{F} \times X \mid x \text { maximizes } f \text { on } X\} .
$$

of $\mathscr{F} \times X$.

As we show in Theorem 1 below, the space $\mathscr{N}$ is homeomorphic to the space $\mathscr{F}$. The main difficulty that the proof has to deal with is the possibility of multiple maxima. To illustrate, let $\overline{\mathscr{F}}$ be a subspace of $\mathscr{F}$ consisting of all strictly concave functions, and let $\overline{\mathscr{N}}$ be the subspace $\mathscr{N} \cap(\overline{\mathscr{F}} \times X)$ of $\mathscr{N}$. Since each function in $\overline{\mathscr{F}}$ has a unique maximum, the natural projection $\bar{\pi}: \overline{\mathscr{N}} \longrightarrow \overline{\mathscr{F}}$ that sends the pair $(f, x)$ to the function $f$ is a one-to-one map. In fact, one can show it is a homeomorphism. It cannot be extended to a homeomorphism between $\mathscr{N}$ and $\mathscr{F}$, however. To see this, observe that $\overline{\mathscr{N}}$ is a dense set of $\mathscr{N}$. It follows that the only continuous extension of $\bar{\pi}$ to the space $\mathscr{N}$ is the natural projection $\pi: \mathscr{N} \longrightarrow \mathscr{F}$ that sends $(f, x)$ to $f$. However, $\pi$ is not an injective mapping.

Given $x \in \mathbb{R}^{k}$, let $l_{x}$ denote the linear function defined by the equation $l_{x}(z)=\langle x, z\rangle$ for all $z \in \mathbb{R}^{k}$. Consider the mapping

$$
\begin{gathered}
\mathscr{N} \stackrel{\phi}{\longrightarrow} \mathscr{F} \\
(f, x) \mapsto f+l_{x} .
\end{gathered}
$$

Theorem 1 The mapping $\phi$ is a homeomorphism. Furthermore, suppose that $\overline{\mathscr{F}}$ is one of the following subspaces of $\mathscr{F}$ : the subspace of linear functions, the subspace of strictly concave functions, or the subspace of $C^{r}$-functions. Let $\bar{N}$ denote the subspace $\mathscr{N} \cap(\overline{\mathscr{F}} \times$ $X)$ of $\mathscr{N}$. Then $\phi \mid \overline{\mathscr{N}}$ is a homeomorphism between $\overline{\mathscr{N}}$ and $\overline{\mathscr{F}}$.

Proof. To see that $\phi$ is a continuous mapping, observe that it extends to a mapping

$$
\mathscr{F} \times X \stackrel{i \times l}{\longrightarrow} \mathscr{F} \times \mathscr{F} \stackrel{+}{\longrightarrow} \mathscr{F},
$$

of $\mathscr{F} \times X$, where $i$ denotes the identity map of $\mathscr{F}, l$ is a map that assigns to each point $x$ of $X$ the linear transformation $l_{x}$, and + denotes the addition map. Since both $l$ and + are continuous with respect to the compact open topology, it follows that $\phi$ is a continuous mapping.

Now we construct the inverse of $\phi$. Let $\bar{f}$ be a real-valued function on $\mathbb{R}^{k}$ defined by $\bar{f}(z)=-\frac{1}{2}\langle z, z\rangle$ for all $z \in \mathbb{R}^{k}$. Let the mapping $\psi$ be defined as follows:

$$
\begin{gathered}
\mathscr{F} \stackrel{\psi}{\longrightarrow} \mathscr{F} \times X \\
f \mapsto\left(f-l_{x}, x\right),
\end{gathered}
$$


where $x$ is the unique maximizer of the strictly concave function $f+\bar{f}$ on the set $X$. To see that the mapping $\psi$ is continuous, let $\mathscr{S} \subset \mathscr{F}$ be the subspace of all strictly concave functions, let $+\bar{f}: \mathscr{F} \longrightarrow \mathscr{S}$ be the mapping that sends the function $f$ into the function $f+\bar{f}$, let $s: \mathscr{S} \longrightarrow X$ be the mapping that assigns to each strictly concave function $f$ the unique maximizer of $f$ on the set $X$, and $l_{-}: X \longrightarrow \mathscr{F}$ be the map that sends each point $x$ of $X$ into the linear transformation $-l_{x}$. The mappings $+\bar{f}, s$ and $l_{-}$are continuous with respect to the compact open topology. Now the component of $\psi$ that maps to $X$ can be written as a composition

$$
\mathscr{F} \stackrel{+\bar{f}}{\longrightarrow} \mathscr{S} \stackrel{s}{\longrightarrow} X
$$

Similarly, the component of $\psi$ that maps to $\mathscr{F}$ is the composite map

$$
\mathscr{F} \stackrel{(i,+\bar{f})}{\longrightarrow} \mathscr{F} \times \mathscr{S} \stackrel{i \times s}{\longrightarrow} \mathscr{F} \times X \stackrel{i \times l_{-}}{\longrightarrow} \mathscr{F} \times \mathscr{F} \stackrel{+}{\longrightarrow} \mathscr{F} .
$$

It follows that $\psi$ is a continuous mapping.

Now we show that $\psi$ maps into the set $\mathscr{N}$. Since the function $(f+\bar{f})$ attains its maximum on the set $X$ at the point $x$, its subdifferential at $x$ has a non-empty intersection with the normal cone $N_{x}[X]$ to the set $X$ at the point $x$. By direct computation, the subdifferential of the function $(f+\bar{f})$ at point $x$ coincides with that of the function $\left(f-l_{x}\right)$ :

$$
\partial(f+\bar{f})(x)=\partial f(x)-\{x\}=\partial\left(f-l_{x}\right)(x) .
$$

This implies that the function $\left(f-l_{x}\right)$ attains its maximum on the set $X$ at the point $x$, as desired.

Clearly, $\phi \circ \psi$ is the identity map on the set $\mathscr{F}$. To see that $\psi \circ \phi$ is the identity on $\mathscr{N}$, let $(f, x)$ be a point in $\mathscr{N}$. Then $(\psi \circ \phi)(f, x)=\left(f+l_{x}-l_{\bar{x}}, \bar{x}\right)$, with $\bar{x}$ being a point of $X$ where the function $\left(f+l_{x}+\bar{f}\right)$ attains its maximum. Since the function $f$ attains its maximum on $X$ at the point $x$, the subdifferential $\partial f(x)$ of $f$ at $x$ has a point in common with the set $N_{x}[X]$. A direct computation shows that

$$
\partial\left(f+l_{x}+\bar{f}\right)(x)=\partial f(x)
$$

implying that the function $\left(f+l_{x}+\bar{f}\right)$ attains its maximum on $X$ at the point $x$. Therefore $x=\bar{x}$, and $(\psi \circ \phi)(f, x)=(f, x)$, as desired. Thus we have proved that $\phi$ is a homeomorphism, with $\psi$ being its inverse.

Finally, observe that the mapping $\phi$ preserves linearity, strict concavity, and $C^{r}$ differentiability of the functions, and so does $\psi$. The second part of the theorem follows.

\section{The structure theorem of Kohlberg and Mertens}

Let $n$ be a positive integer, and let $N$ denote the set of integers $\{1, \ldots, n\}$. For each $i \in N$, let $k^{i}$ be a positive integer, and let $k=\sum_{i \in N} k^{i}$ and $p=\times_{i \in N} k^{i}$. Let $\Delta^{i}$ denote the unit simplex in $\mathbb{R}^{k^{i}}$, and let $J^{i}$ denote the set of vertices of $\Delta^{i}$. Let $\Delta$ denote the product space 
$\Delta^{1} \times \cdots \times \Delta^{n}$, and let $J$ denote the product $J^{1} \times \cdots \times J^{n}$. The typical elements of $\mathbb{R}^{k^{i}}$ are denoted by $x^{i}$ or $z^{i}$, the typical element of the product space $\mathbb{R}^{k}=\mathbb{R}^{k^{1}} \times \cdots \times \mathbb{R}^{k^{n}}$ is denoted by $x=\left(x^{1}, \ldots, x^{n}\right)$, the typical element of $J^{i}$ is $j^{i}$ and the typical element of $J$ is $j=\left(j^{1}, \ldots, j^{n}\right)$. Given $x^{i} \in \mathbb{R}^{k^{i}}$ and $j^{i} \in J^{i}$, we let $x_{j^{i}}^{i}$ denote the component of the vector $x^{i}$ that corresponds to $j^{i}$.

In this section we consider normal form games where $N$ is the set of players, $\Delta^{i}$ is the strategy set of player $i$ and the payoff function $u^{i}: \mathbb{R}^{k} \longrightarrow \mathbb{R}$ of player $i$ is a multilinear function. A function $u^{i}$ is said to be multilinear if $u^{i}\left(\cdot, z^{-m}\right): \mathbb{R}^{k^{m}} \longrightarrow \mathbb{R}$ is a linear function for every $z \in \mathbb{R}^{k}$ and every $m \in N$. Recall that a multilinear function $u^{i}$ can be written in the form

$$
u^{i}(x)=\sum_{j \in J}\left[\underset{m=1}{\stackrel{n}{\times}} x_{j^{m}}^{m}\right] u^{i}(j),
$$

and is therefore entirely determined by its values on the set $J$. Thus the space of multilinear payoff functions of player $i$ can be identified with the $p$-dimensional Euclidean space, in a natural way, and the space of normal form games with multilinear payoffs can be identified with $\mathbb{R}^{n p}$.

A Nash equilibrium of the game $u=\left(u^{1}, \ldots, u^{n}\right) \in \mathbb{R}^{n p}$ is a strategy combination $x \in \Delta$ such that for each player $i$ the strategy $x^{i}$ maximizes the function $u^{i}\left(\cdot, x^{-i}\right): \mathbb{R}^{k^{i}} \longrightarrow \mathbb{R}$ on the set $\Delta^{i}$. The Nash equilibrium correspondence assigns to the game $u$ in $\mathbb{R}^{n p}$ the set of Nash equilibria of the game $u$. Its graph is the subspace of $\mathbb{R}^{n p} \times \Delta$ given by

$$
\mathscr{G}=\left\{(u, x) \in \mathbb{R}^{n p} \times \Delta \mid \begin{array}{c}
x \text { is a Nash equilibrium } \\
\text { of the game } u
\end{array}\right\} .
$$

Theorem 2 (Kohlberg and Mertens, 1986) The space $\mathscr{G}$ is homeomorphic to $\mathbb{R}^{n p}$.

The homeomorphism used by Kohlberg and Mertens is defined as a mapping

$$
\begin{gathered}
\mathscr{G} \stackrel{\gamma}{\longrightarrow} \mathbb{R}^{n p} \\
\left(\left(u^{i}\right)_{i=1}^{n}, x\right) \mapsto\left(\bar{u}^{i}\right)_{i=1}^{n},
\end{gathered}
$$

where $\bar{u}^{i}$ is an element of $\mathbb{R}^{p}$ given by the equation

$$
\begin{aligned}
\bar{u}^{i}(j)= & u^{i}(j)-\sum_{l^{-i} \in J^{-i}}\left[\underset{m \in N \backslash\{i\}}{\times} \frac{1}{k^{m}}\right] u^{i}\left(j^{i}, l^{-i}\right)+ \\
& +x_{j^{i}}^{i}+\sum_{l^{-i} \in J^{-i}}\left[\underset{m \in N \backslash\{i\}}{\times} x_{l^{m}}^{m}\right] u^{i}\left(j^{i}, l^{-i}\right) .
\end{aligned}
$$

It is helpful to have an expression for the value of $\bar{u}^{i}$ at an arbitrary point of $\mathbb{R}^{k}$. For each player $i$, let $\mathbf{1}^{i}$ be the vector of ones in $\mathbb{R}^{k^{i}}$ and let $a^{i}$ be the barycenter of $\Delta^{i}$. That is, $a_{j^{i}}^{i}=\frac{1}{k^{i}}$ for all $j^{i} \in J^{i}$. Then for a point $z$ of $\mathbb{R}^{k}$, we have

$$
\bar{u}^{i}(z)=u^{i}(z)+\left[u^{i}\left(z^{i}, x^{-i}\right)-u^{i}\left(z^{i}, a^{-i}\right)+\left\langle z^{i}, x^{i}\right\rangle\right] \underset{m \in N \backslash\{i\}}{\times}\left\langle\mathbf{1}^{m}, z^{m}\right\rangle .
$$

The product after the square brackets is equal to one whenever $z$ is an element of $\Delta$. 


\section{The general structure theorem}

In this section we study a richer class of games than the class of normal form games introduced in the previous section. Now the payoff function of player $i$ need not be a multilinear function, but is only required to be continuous and concave in player $i$ 's own strategies. Moreover, we allow the strategy set of each player to be a non-empty compact convex subset of Euclidean space. In this setting we prove that the graph of the Nash equilibrium correspondence is homeomorphic to the space of games. We refine this result by demonstrating that, if the player's strategy sets do not contain the zero vector, then there exists a homeomorphism between the graph of the Nash correspondence and the space of games that preserves the multilinearity in the payoff functions. This allows us to derive Theorem 2 as a corollary to our general structure theorem.

Let the strategy set $X^{i}$ of player $i \in N$ be a non-empty compact convex set of $\mathbb{R}^{k^{i}}$. Let $X$ denote the product space $X^{1} \times \cdots \times X^{n}$. Let $\mathscr{F}^{i}$ be the set of payoff functions $u^{i}$ of player $i$ defined on the product space $\mathbb{R}^{k}=\mathbb{R}^{k^{1}} \times \cdots \times \mathbb{R}^{k^{n}}$ such that $u^{i}$ is continuous on $\mathbb{R}^{k}$ and concave on $\mathbb{R}^{k^{i}}$. We endow $\mathscr{F}^{i}$ with the compact open topology and we let $\mathscr{F}$ denote the product space $\mathscr{F}^{1} \times \cdots \times \mathscr{F}^{n}$. The graph of the Nash equilibrium correspondence is the subspace of $\mathscr{F} \times X$ given by

$$
\mathscr{N}=\left\{(u, x) \in \mathscr{F} \times X \mid \begin{array}{c}
x \text { is a Nash equilibrium } \\
\text { of the game } u
\end{array}\right\} .
$$

Definition 1 For each player $i$, let $a^{i}$ be a point in $\mathbb{R}^{k^{i}}$. Let $t^{i}: X^{i} \times \mathbb{R}^{k^{i}} \longrightarrow \mathbb{R}^{k^{i}}$ be a continuous mapping such that for each point $x^{i}$ in $X^{i}$ the mapping $t^{i}\left(x^{i}, \cdot\right)=t_{x^{i}}^{i}$ : $\mathbb{R}^{k^{i}} \longrightarrow \mathbb{R}^{k^{i}}$ is a homeomorphism that carries the point $a^{i}$ to $x^{i}$. Let $r^{i}: X^{i} \times \mathbb{R}^{k} \longrightarrow \mathbb{R}$ be a continuous mapping such that for each point $x^{i}$ in $\mathbb{R}^{k^{i}}$ the function $r^{i}\left(x^{i}, \cdot\right)=r_{x^{i}}^{i}: \mathbb{R}^{k} \longrightarrow \mathbb{R}$ belongs to the space $\mathscr{F}^{i}$ and satisfies the equation $r_{x^{i}}^{i}\left(z^{i}, a^{-i}\right)=\left\langle x^{i}, z^{i}\right\rangle$ for all $z^{i} \in \mathbb{R}^{k^{i}}$. Given an $x \in X$, let $p_{x}^{i}: \mathbb{R}^{k} \longrightarrow \mathbb{R}^{k}$ denote the homeomorphism

$$
t_{x^{1}}^{1} \times \cdots \times t_{x^{i-1}}^{i-1} \times i d \times t_{x^{i+1}}^{i+1} \times \cdots \times t_{x^{n}}^{n},
$$

where $i d$ is the identity map on $\mathbb{R}^{k^{i}}$. Given the mappings $t^{i}$ and $r^{i}$, define the mapping $\phi$ as follows:

$$
\begin{gathered}
\mathscr{N} \stackrel{\phi}{\stackrel{F}{\longrightarrow}} \mathscr{F} \\
\left(\left(u^{i}\right)_{i=1}^{n}, x\right) \stackrel{\left(u^{i} \circ p_{x}^{i}+r_{x^{i}}^{i}\right)_{i=1}^{n} .}{ } .
\end{gathered}
$$

Observe that, when $n=1$ (the one-player case), the mapping $\phi$ as defined above coincides with the mapping introduced in Section 3. The freedom that one has in the choice of the mappings $t^{i}$ and $r^{i}$ will be exploited later to construct the mapping $\phi$ that preserves the multilinearity in the payoff functions. Now we state our basic result.

Theorem 3 The mapping $\phi$ is a homeomorphism. 
Proof. Define the mapping $\psi$ as follows:

$$
\begin{gathered}
\mathscr{F} \stackrel{\psi}{\longrightarrow} \mathscr{F} \times X \\
\left(u^{i}\right)_{i=1}^{n} \mapsto\left(\left(\left[u^{i}-r_{x^{i}}^{i}\right] \circ\left[p_{x}^{i}\right]^{-1}\right)_{i=1}^{n}, x\right),
\end{gathered}
$$

where $x^{i}$ for each $i$ is the unique maximizer of the strictly concave function

$$
z^{i} \mapsto u^{i}\left(z^{i}, a^{-i}\right)-\frac{1}{2}\left\langle z^{i}, z^{i}\right\rangle
$$

over the set $X^{i}$. Note that both functions $\left[u^{i} \circ p_{x}^{i}+r_{x^{i}}^{i}\right]$ and $\left[u^{i}-r_{x^{i}}^{i}\right] \circ\left[p_{x}^{i}\right]^{-1}$ belong to the set $\mathscr{F}^{i}$ whenever $u^{i}$ does, because $r_{x^{i}}$ belongs to $\mathscr{F}^{i}$, and because $p_{x}^{i}$ is a product of the homeomorphisms on $\mathbb{R}^{k^{j}}$ for all $j \neq i$ and the identity map on $\mathbb{R}^{k^{i}}$.

The continuity of the mappings $\phi$ and $\psi$ is straightforward to verify. The argument follows the pattern set by the proof of Theorem 1 .

We show that $\psi$ maps to the set $\mathscr{N}$. Let $u$ be a point in $\mathscr{F}$, and suppose that $\psi(u)=(\bar{u}, x)$. We must show that $x^{i}$ maximizes the function $\bar{u}^{i}\left(\cdot, x^{-i}\right)$ on the set $X^{i}$. Since the point $x^{i}$ of $X^{i}$ is determined as the maximum of the function $u^{i}\left(z^{i}, a^{-i}\right)-\frac{1}{2}\left\langle z^{i}, z^{i}\right\rangle$, we know that

$$
\left[\partial u^{i}\left(x^{i}, a^{-i}\right)-\left\{x^{i}\right\}\right] \cap N_{x^{i}}\left[X^{i}\right]
$$

is a non-empty set. As the homeomorphism $p_{x}^{i}$ carries the point $\left(z^{i}, a^{-i}\right)$ to $\left(z^{i}, x^{-i}\right)$, we have the following sequence of equations:

$$
\begin{aligned}
\bar{u}^{i}\left(z^{i}, x^{-i}\right) & =\left[u^{i}-r_{x^{i}}^{i}\right] \circ\left[p_{x}^{i}\right]^{-1}\left(z^{i}, x^{-i}\right) \\
& =\left[u^{i}-r_{x^{i}}^{i}\right]\left(z^{i}, a^{-i}\right) \\
& =u^{i}\left(z^{i}, a^{-i}\right)-\left\langle x^{i}, z^{i}\right\rangle,
\end{aligned}
$$

for all $z^{i} \in \mathbb{R}^{k^{i}}$. This implies that

$$
\partial \bar{u}^{i}\left(x^{i}, x^{-i}\right)=\partial u^{i}\left(x^{i}, a^{-i}\right)-\left\{x^{i}\right\} .
$$

Thus $x^{i}$ maximizes $\bar{u}^{i}\left(\cdot, x^{-i}\right)$ over the set $X^{i}$, as desired.

Clearly, $\phi \circ \psi$ is the identity map on $\mathscr{F}$. To see that $\psi \circ \phi$ is the identity map on $\mathscr{N}$, let $(u, x)$ be a point in $\mathscr{N}$. Then $(\psi \circ \phi)(u, x)=(\bar{u}, \bar{x})$, where $\bar{u}^{i}=\left[u^{i} \circ p_{x}^{i}+r_{x^{i}}^{i}-r_{\bar{x}^{i}}^{i}\right] \circ\left[p_{\bar{x}}^{i}\right]^{-1}$, and $\bar{x}^{i}$ is a unique point of $X^{i}$ where the function

$$
\begin{aligned}
z^{i} \mapsto \quad & \left(u^{i} \circ p_{x}^{i}+r_{x^{i}}^{i}\right)\left(z^{i}, a^{-i}\right)-\frac{1}{2}\left\langle z^{i}, z^{i}\right\rangle= \\
& u^{i}\left(z^{i}, x^{-i}\right)+\left\langle x^{i}, z^{i}\right\rangle-\frac{1}{2}\left\langle z^{i}, z^{i}\right\rangle
\end{aligned}
$$

attains its maximum. As a direct computation shows, its subdifferential at $x^{i}$ equals $\partial u^{i}\left(x^{i}, x^{-i}\right)$. Since $x^{i}$ is the maximum of the function $u^{i}\left(\cdot, x^{-i}\right)$ on $X^{i}$, we know that $\partial u^{i}\left(x^{i}, x^{-i}\right) \cap N_{x^{i}}\left[X^{i}\right]$ is a non-empty set. Thus $\bar{x}^{i}=x^{i}$ for each player $i$, and $\bar{u}=u$, as desired. We have thus proved that $\phi$ is a homeomorphism, with $\psi$ being its inverse. 
Now that we have a family of homeomorphisms between the spaces $\mathscr{N}$ and $\mathscr{F}$, we can identify those homeomorphisms that preserve some natural properties of the payoff functions such as multilinearity, differentiability, and linearity (strict concavity) of the payoff function $u^{i}$ in player $i$ 's own strategies. We say that the payoff function $u^{i}$ is linear (strictly concave) in $z^{i}$ if $u^{i}\left(\cdot, z^{-i}\right): \mathbb{R}^{k^{i}} \rightarrow \mathbb{R}$ is a linear (strictly concave) function for each $z \in \mathbb{R}^{k}$.

Theorem 4 below provides a homeomorphism between $\mathscr{N}$ and $\mathscr{F}$ that preserves differentiability of the payoff functions and, for each player $i$, linearity and strict concavity of the payoff function $u^{i}$ in $z^{i}$. In Theorem 5 we establish the existence of a homeomorphism between $\mathscr{N}$ and $\mathscr{F}$ that preserves multilinearity in the payoff functions, and derive Theorem 2 as a corollary.

Consider the mapping

$$
\begin{gathered}
\mathscr{N} \stackrel{\eta}{\longrightarrow} \mathscr{F} \\
\left(\left(u^{i}\right)_{i=1}^{n}, x\right) \mapsto\left(\bar{u}^{i}\right)_{i=1}^{n}, \quad \text { where } \\
\bar{u}^{i}(z)=u^{i}\left(z^{i}, z^{-i}-a^{-i}+x^{-i}\right)+\left\langle z^{i}, x^{i}\right\rangle .
\end{gathered}
$$

Theorem 4 The mapping $\eta$ is a homeomorphism. Furthermore, let $\overline{\mathscr{F}}^{i}$ be one of the following subspaces of $\mathscr{F}^{i}$ : the subspace of $C^{r}$-functions, the subspace of functions $u^{i}$ that are linear in $z^{i}$, or the subspace of functions $u^{i}$ that are strictly concave in $z^{i}$. Let $\overline{\mathscr{F}}=$ $\overline{\mathscr{F}}^{1} \times \cdots \times \overline{\mathscr{F}}^{n}$, and let $\overline{\mathscr{N}}$ denote the subspace $\mathscr{N} \cap(\overline{\mathscr{F}} \times X)$ of $\mathscr{N}$. Then the mapping $\eta \mid \overline{\mathscr{N}}$ is a homeomorphism between $\overline{\mathscr{N}}$ and $\overline{\mathscr{F}}$.

Proof. The mapping $\eta$ coincides with $\phi$, provided that the mappings $t^{i}: X^{i} \times \mathbb{R}^{k^{i}} \longrightarrow \mathbb{R}^{k^{i}}$ and $r^{i}: X^{i} \times \mathbb{R}^{k} \longrightarrow \mathbb{R}$ are given by the equations $t^{i}\left(x^{i}, z^{i}\right)=z^{i}-a^{i}+x^{i}$ and $r^{i}\left(x^{i}, z^{i}, z^{-i}\right)=$ $\left\langle x^{i}, z^{i}\right\rangle$, respectively. Thus $\eta$ is a homeomorphism and its inverse is the mapping

$$
\begin{gathered}
\mathscr{F} \longrightarrow \mathscr{N} \\
\left(\bar{u}^{i}\right)_{i=1}^{n} \mapsto\left(\left(u^{i}\right)_{i=1}^{n}, x\right), \quad \text { where } \\
u^{i}(z)=\bar{u}^{i}\left(z^{i}, z^{-i}+a^{-i}-x^{-i}\right)-\left\langle z^{i}, x^{i}\right\rangle .
\end{gathered}
$$

It is easy to see that both $\eta$ and its inverse carry $C^{r}$-functions to $C^{r}$-functions. If the function $u^{i}$ is linear (strictly concave) in $z^{i}$, then so is the function $\bar{u}^{i}$, and conversely. The result follows.

Now we would like to have a homeomorphism between $\mathscr{N}$ and $\mathscr{F}$ that carries multilinear functions to multilinear functions. The homeomorphism $\eta$ does not possess this property: while $\eta$ preserves linearity of $u^{i}$ in player $i$ 's strategies, it does not preserve linearity of $u^{i}$ in strategies of player $i$ 's opponents. For the homeomorphism $\phi$ to preserve the multilinearity, it suffices that $t_{x^{i}}^{i}$ be a linear map, and $r_{x^{i}}^{i}$ be a multilinear function for all $x^{i}$. The existence of $t^{i}$ and $r^{i}$ with these properties is asserted by Propositions 1 and 2 below. 
Proposition 1 Suppose that the set $X^{i}$ does not contain the zero vector. Let $a^{i}$ be a nonzero vector of $\mathbb{R}^{k^{i}}$. Then there exists a continuous mapping $t^{i}: X^{i} \times \mathbb{R}^{k^{i}} \longrightarrow \mathbb{R}^{k^{i}}$ such that for each point $x^{i}$ in $X^{i}$ the mapping $t^{i}\left(x^{i}, \cdot\right)=t_{x^{i}}^{i}: \mathbb{R}^{k^{i}} \longrightarrow \mathbb{R}^{k^{i}}$ is a linear isomorphism that carries the point $a^{i}$ to $x^{i}$.

Proof. Choose the vectors $d^{1}, \ldots, d^{k^{i}-1}$ in $\mathbb{R}^{k^{i}}$ so that the $\left(k^{i} \times k^{i}\right)$-dimensional matrix $A=\left[a^{i}, d^{1}, \ldots, d^{k^{i}-1}\right]$ is non-singular. By the separating hyperplane theorem, there is a vector $s$ in $\mathbb{R}^{k^{i}}$ such that $\left\langle s, x^{i}\right\rangle>0$ for all $x^{i} \in X^{i}$. Let $b^{1}, \ldots, b^{k^{i}-1}$ be a basis of the linear subspace of $\mathbb{R}^{k^{i}}$ orthogonal to the vector $s$. Given an $x^{i} \in X^{i}$, let $M_{x^{i}}$ denote the $\left(k^{i} \times k^{i}\right)$-dimensional matrix $\left[x^{i}, b^{1}, \ldots, b^{k^{i}-1}\right]$. Observe that $M_{x^{i}}$ is a non-singular matrix. Let $T_{x^{i}}=M_{x^{i}} A^{-1}$ be the matrix representing the linear transformation $t_{x^{i}}^{i}$. The matrix $T_{x^{i}}$ is non-singular, it depends continuously on $x^{i}$, and $T_{x^{i}} a^{i}=x^{i}$. The result follows.

If the set $X^{i}$ contains the zero vector, the assertion of Proposition 1 is invalid, for there is no linear isomorphism of $\mathbb{R}^{k^{i}}$ that carries a non-zero vector $a^{i}$ to zero.

Proposition 2 For each $i$ let $a^{i}$ be a strictly positive vector of $\mathbb{R}^{k^{i}}$. Then there exists a continuous mapping $r^{i}: \mathbb{R}^{k^{i}} \times \mathbb{R}^{k} \longrightarrow \mathbb{R}$ such that for each point $x^{i}$ in $\mathbb{R}^{k^{i}}, r^{i}\left(x^{i}, \cdot\right)=r_{x^{i}}^{i}$ : $\mathbb{R}^{k} \longrightarrow \mathbb{R}$ is a multilinear function satisfying $r_{x^{i}}^{i}\left(z^{i}, a^{-i}\right)=\left\langle x^{i}, z^{i}\right\rangle$ for all $z^{i} \in \mathbb{R}^{k^{i}}$.

Proof. For each $x^{i}$ in $\mathbb{R}^{k^{i}}$ we define the multilinear function $r_{x^{i}}^{i}: \mathbb{R}^{k} \longrightarrow \mathbb{R}$ by specifying its action on the set $J$, as follows:

$$
r_{x^{i}}^{i}(j)=\frac{x_{j^{i}}^{i}}{\underset{\substack{\times \\ m \in N \backslash\{i\}}}{j_{j^{m}}^{m}}} .
$$

Since $r_{x^{i}}^{i}(j)$ depends continuously on $x^{i}$, the result follows.

Theorem 5 Suppose that for each player $i$ the set $X^{i}$ does not contain the zero vector. Let $\mathscr{F}^{* i}$ be the subspace of multilinear functions. Let $\mathscr{F}^{*}=\mathscr{F}^{* 1} \times \cdots \times \mathscr{F}^{* n}$, and let $\mathscr{N}^{*}$ denote the subspace $\mathscr{N} \cap\left(\mathscr{F}^{*} \times X\right)$ of $\mathscr{N}$. Then there exists a homeomorphism $\phi: \mathscr{N} \longrightarrow \mathscr{F}$ such that $\phi \mid \mathscr{N}^{*}$ is a homeomorphism between $\mathscr{N}^{*}$ and $\mathscr{F}^{*}$.

Proof. Let $\phi$ be as in Definition 1, with $t^{i}$ and $r^{i}$ given by Propositions 1 and 2 . Then both $\phi$ and its inverse $\psi$ carry multilinear functions to multilinear functions.

Corollary 1 The space $\mathscr{G}$ is homeomorphic with $\mathbb{R}^{n p}$.

Proof. The subspace $\mathscr{F}^{i *}$ of multilinear payoff functions of player $i$ is homeomorphic to the Euclidean space $\mathbb{R}^{p}$, the space $\mathscr{F}^{*}$ is homeomorphic to $\mathbb{R}^{p n}$, and the space $\mathscr{N}^{*}$ is homeomorphic to $\mathscr{G}$. The corollary follows at once from Theorem 5 . 


\section{Conclusions}

This paper provides a topological characterization of the graph of the Nash equilibrium correspondence in a very general setup. We consider normal form games where the strategy set of each player is a non-empty compact convex set of the Euclidean space, and the payoff function of player $i$ is continuous and concave in player $i$ 's own strategies.

Our basic result states that the graph $\mathscr{N}$ of the Nash equilibrium correspondence is homeomorphic to the space $\mathscr{F}$ of games, as parameterized by the payoff functions. This basic result is supplemented by two important refinements, demonstrating the existence of homeomorphisms between $\mathscr{N}$ and $\mathscr{F}$ that are, in a certain sense, well-behaved.

The first of the refinements shows that there exists a homeomorphism between $\mathscr{N}$ and $\mathscr{F}$ that preserves differentiability of the payoff function of each player $i$ in all variables and linearity and strict concavity in player $i$ 's own strategies.

The second refinement applies whenever the strategy sets do not contain the zero vectors. In this case, there exists a homeomorphism between $\mathscr{N}$ and $\mathscr{F}$ that preserves multilinearity in the payoff functions. As a corollary, we show that the graph of the Nash equilibrium correspondence restricted to the subspace of normal form games with multilinear payoffs is homeomorphic to a finite-dimensional Euclidean space. This is a known result due to Kohlberg and Mertens [6].

\section{References}

[1] Yves Balasko. Economic Equilibrium and Catastrophe Theory: An Introduction. Econometrica, 46: 557-569, 1978.

[2] Lawrence E. Blume and William Zame. The Algebraic Geometry of Perfect and Sequential Equilibrium. Econometrica, 62: 783-794, 1994.

[3] Stefano Demichelis and Fabrizio Germano. Some Consequences of the Unknottedness of the Walras Correspondence. Journal of Mathematical Economics, 34: $537-45,2000$.

[4] Stefano Demichelis and Fabrizio Germano. On (Un)knots and Dynamics in Games. Games and Economic Behavior, 41: 46-60, 2002.

[5] Srihari Govindan and Robert Wilson. Direct Proofs of Generic Finiteness of Nash Equilibrium Outcomes. Econometrica, 69: 765-769, 2001.

[6] Elon Kohlberg and Jean-François Mertens. On the Strategic Stability of Equilibria. Econometrica, 54: 1003-1037, 1986.

[7] R. Tyrrell Rockafellar. Convex Analysis. Princeton University Press, Princeton, New Jersey, 1997.

[8] Yuqing Zhou. Genericity Analysis on the Pseudo-Equilibrium Manifold. Journal of Economic Theory, 73: 79-92, 1997. 